\title{
ABUNDANCIA DE Amblyrhynchus cristatus EN EL NIÑO (97-98) Y LA NIÑA (01-02) EN SANTA CRUZ, GALÁPAGOS
}

\section{ABUNDANCE OF Amblyrhynchus cristatus IN EL NIÑO (97-98) AND LA NIÑA (01-02) IN SANTA CRUZ, GALAPAGOS}

\author{
Alizon Llerena ${ }^{1}$, Cruz Márquez ${ }^{2}$, Howard L. Snell ${ }^{3}$ y Alberto Jaramillo ${ }^{4}$
}

\section{Resumen}

La iguana marina (Amblyrhynchus cristatus Bell, 1825) es una especie endémica de Galápagos que forrajea en el mar (Bartholomew \& Vleck, 1979). Estudios científicos han mostrado que el Evento El Niño Oscilación Sur (ENOS) produce impactos negativos en la fauna de las islas (Wikelski \& Corinna, 2000; Romero \& Wikelski, 2001) y en 1997 la Fundación Charles Darwin (FCD) establece un sitio de muestreo permanente al Sur de la isla Santa Cruz, entre Punta Núñez y el muelle de la FCD, para obtener información científica y extrapolarla para el conocimiento del efecto de El Niño en otras poblaciones de iguanas marinas del archipiélago y su recuperación en los subsiguientes años. Se registró la abundancia en una transecta de $6.1 \mathrm{~km}$ dividida en estaciones de $100 \mathrm{~m}$ (61 estaciones) durante un día cada mes. ANOVA y t-test fueron usados para comparar las abundancias en El Niño (1997-98) y La Niña (2001-02), usando solamente los datos del segundo semestre de cada año; y estas fueron diferentes $(\mathrm{t}=4.349 ; \mathrm{gl}=5 ; \mathrm{P}<0.004)$. Al comparar la abundancia en los estratos poblacionales de A. cristatus, los machos adultos fueron menos abundante durante El Niño con respecto a La Niña ( $\mathrm{t}=2.195 ; \mathrm{P}<0.0039)$. En hembras los promedios de abundancia no difieren $(\mathrm{t}=0.171 \mathrm{P}>0.44)$ mientras en juveniles la diferencia es significativa, notándose la recuperación en La Niña $(\mathrm{t}=7.377 ; \mathrm{P}<0.0004)$. Los resultados indican que las iguanas marinas, luego de un evento El Niño, tardan aproximadamente 4 años para recuperarse.

Palabras Calves: Abundancia, El Niño-La Niña, Amblyrhynchus cristatus, Santa Cruz, Galápagos.

\begin{abstract}
The marine iguana (Amblyrhynchus cristatus Bell, 1825) is an endemic species in Galapagos that forages in the sea (Bartholomew \& Vleck, 1979). Scientific studies of marine iguanas have shown that El Niño Southern Oscillation events (ENSO) produce negative impacts on the fauna of the isles (Wikelski \& Corinna, 2000; Romero \& Wikelski, 2001) and in 1997 the Charles Darwin Foundation (CDF) established a permanent sampling site in southern Santa Cruz Island, between Punta Núñez and the CDF dock, to obtain scientific information and extrapolate it to understand the effect of El Niño on other marine iguana populations of the archipelago and their recuperation in succeeding years. Abundance was recorded along a $6.1 \mathrm{~km}$ transect divided into sections of 100 $\mathrm{m}$ (61 sections) on one day each month. ANOVA and Student's t-tests were used to compare abundance in El Niño (1997-98) and La Niña years (2001-2002), using only data from the second six months of each year; they differed significantly $(\mathrm{t}=4.349, \mathrm{P}<0.004)$. On comparing the abundance of population classes of $A$. cristatus, adult males were less common during El Niño than during La Niña $(\mathrm{t}=2.195 ; \mathrm{P}<0.0039)$. Among females, abundance means did not differ $(\mathrm{t}=$ $0.171 ; \mathrm{P}>0.44)$, while in juveniles the difference is significant, indicating population recuperation in La Niña $(\mathrm{t}=7.337 ; \mathrm{P}<0.0004)$. The results indicate that marine iguanas, after an El Niño event, take about 4 years to recover.
\end{abstract}

Key Words: Abundante, El Niño-La Niña, Amblyrhynchus cristatus, Santa Cruz, Galapagos.

\section{Introducción}

La iguana marina (Amblyrhynchus cristatus Bell, 1825) pertenece a uno de los dos géneros de iguanas endémicos del Archipiélago de Galápagos (Snell \& Márquez, 2002) y está ampliamente distribuida en las islas, con mayores concentraciones en las islas occidentales (Laurie, 1986). Las iguanas marinas son los únicos lagartos en el mundo que forrajean bajo el mar (Bartholomew \& Vleck, 1979). Son herbívoros que se alimentan de algas inter y submareales a lo largo de las costas rocosas de las islas Galápagos del Ecuador (Wikelski \& Corinna, 2000). Laurie (1984) sugirió que las iguanas marinas son más abundantes en los años de La Niña (años con aguas marinas frías, con temperaturas menores que el promedio normal), cuando las algas verdes y rojas son abundantes como alimento disponible en su dieta preferida, opuesto a los años El Niño o también llamadas ENOS (El Niño - 
Oscilación Sur; años con aguas cálidas y temperaturas mayores de los promedios normales). La abundancia de iguanas marinas son menores cuando ocurre un año de El Niño.

El aumento en las precipitaciones que acompañan a El Niño tiene como resultado una mayor disponibilidad de alimentos para la mayoría de los organismos terrestres de Galápagos, pero en general, la vida marina es la que más sufre de consecuencias agresivas del ambiente (Cayot, 1985). Durante los años de El Niño en Galápagos, las temperaturas de la superficie del mar aumentan de promedios de $18{ }^{\circ} \mathrm{C}$ a un máximo de $32{ }^{\circ} \mathrm{C}$, debido a que se interrumpen las corrientes frías, y con ello los afloramientos ricos en nutrientes.

Los eventos ENOS se repiten en intervalos de 3 a 7 años, pero luego de 1990 fueron más continuos. El índice de la Oscilación del Sur, en síntesis, muestra la gravedad de El Niño, y fue positivo (indicativa de condiciones de La Niña) durante los años 1990-1995 y negativo (indicativa de condiciones de El Niño) en 1997-1998 cuando las temperaturas superficiales del mar aumentaron en promedio por la interrupción de las corrientes frías (Wikelski et al., 1993).

La escasez de alimento en los años de El Niño hace que las iguanas marinas mueran produciéndose un descenso en su abundancia (Cooper \& Laurie, 1987; Castillo et al., no publicada). De ahí resalta la importancia de un monitoreo continuo sobre la población de iguanas marinas. En 1997 la FCD (Fundación Charles Darwin) empezó un programa de monitoreo a largo plazo con el fin de conocer la interacción entre estos organismos y el ambiente en función del fenómeno de El Niño con las repercusiones posibles para su conservación en tiempo y espacio.

\section{Materiales y métodos}

El área de estudio se ubica al sur de la Isla Santa Cruz hacia el oeste del Archipiélago de Galápagos y tiene una extensión de $6.1 \mathrm{~km}$, que equivalen al 3.5\% de los $173 \mathrm{~km}$ que constituyen el perímetro total de la isla. Consta de 61 estaciones de $100 \mathrm{~m}$ cada una, desde la entrada de Punta Núñez con la estación No.1 $\left(0.74588^{\circ}\right.$ lat. S y $90.25537^{\circ}$ long. O) hasta la estación No. $61\left(0.74339^{\circ}\right.$ lat S. y $90.30470^{\circ}$ long. O) en el muelle de la Estación Científica Charles Darwin (Figura 1).

Laurie (1984) describe el clima local como biestacional: de Enero a Mayo es la estación caliente con lluvia representativa y de Junio a Diciembre es frío y frecuentemente nublado con persistente y muy ligera llovizna. El suelo está constituido principalmente por arena orgánica (conchilla, restos de erizos y coral) y rocas de origen volcánico. Aproximadamente $4 \mathrm{~km}$ de la transecta está formada por un acantilado cuya altura va desde los $3 \mathrm{~m}$ hasta los $33 \mathrm{~m}$ de altura. La vegetación terrestre está conformada por manglar y plantas de borde costero (Sesuvium Molina, 1975) mientras que la flora marina está constituida por macroalgas rojas (Rodophyta), verdes (Chlorophyta) y grises (Phaeophyta).

A partir de enero de 1998, durante el último día de cada mes se realizó el trabajo de campo; el traslado al lugar se hizo en un bote con motor fuera de borda (panga) el acceso a la zona tuvo que hacerse por lo menos una hora antes o después de la marea alta, por ser este un sector muy peligroso de la cosa. Para la medición de la abundancia de $A$. cristatus, se caminó a lo largo de las 61 estaciones desde las primeras horas de la mañana (07H00) cuando la actividad de las iguanas marinas es mayor. Partiendo desde la estación No.1, se contó todas las iguanas que están al paso en un área de más o menos $8 \mathrm{~m}$ a cada lado de la transecta lineal, por lo que se está calculando una abundancia relativa con respecto al espacio observado. Fue necesaria la utilización de binoculares que facilitó la observación de los individuos, sobre todo en los acantilados más altos. Los datos obtenidos se anotaron en formularios que categorizan a las iguanas por tamaño y sexo. Además se registró el estado del tiempo y la condición de la marea que influye en la presencia o ausencia de los animales. El conteo total duró aproximadamente 8 horas, tiempo que se tarda en recorrer caminado los $6.1 \mathrm{~km}$.

Por ausencia de información continua en el primer semestre de los años 1997 y 1998 se decidió incluir en el análisis únicamente los datos del segundo semestre de los años de El Niño (1997-1998) y de La Niña (2001-2002), para que la información pueda ser comparativa y los resultados sean confiables.

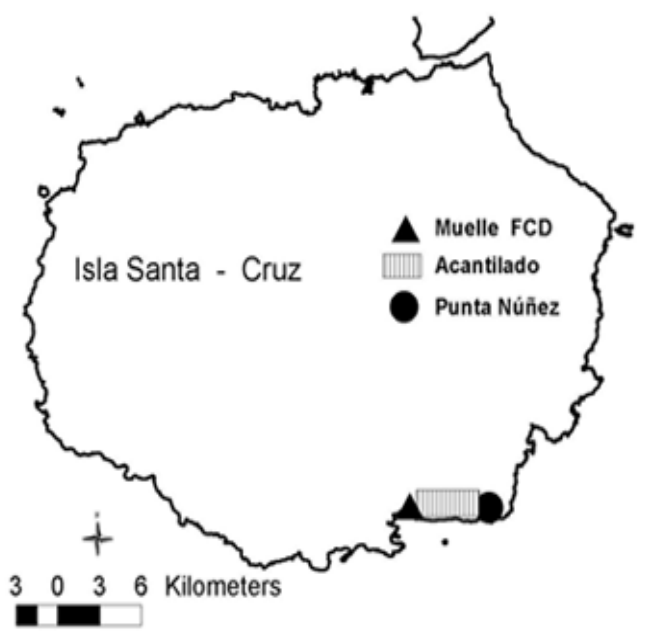

Figura 1. El área del estudio se extiende por la costa desde Punta Núñez hasta el muelle de la ECCD.

\section{Resultados}

Los promedios de abundancia de $A$. cristatus durante El Niño (36.42) comparadas con las de La Niña (44.74) fueron significativamente diferentes $(t=$ 
$4.349 ; \mathrm{gl}=5 ; \mathrm{P}<0.004) ;$ con mucha más relevancia se observa que difieren los promedios de 1998 (24.23), El Niño, con los del 2002 (51.55), La Niña (Figura 2).

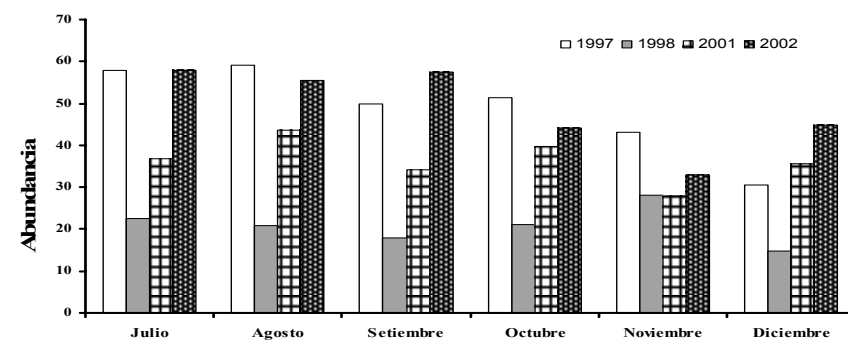

Figura 2. Abundancia promedio de A. cristatus, registrada a lo largo de una transecta de $6.1 \mathrm{~km}$ establecidos en 61 estaciones georeferenciadas entre Punta Núñez y el Muelle de la Estación Científica Charles Darwin, durante El Niño (1997-1998) y La Niña (2001-2002).

Los promedios mensuales de abundancia de $A$. cristatus en El Niño fueron más variables (coeficiente de varianción $1997=0.84 ; 1998=0.93)$ con relación a los de La Niña (coeficiente de variación 2001=0.98; $2002=0.96$ ), mostrando una abundancia por estación de muestreo poco variable en El Niño y muy variable en La Niña ( $\mathrm{F}=15.195 ; \mathrm{gl}=5 ; \mathrm{P}<0.0025$ Figura 3$)$.

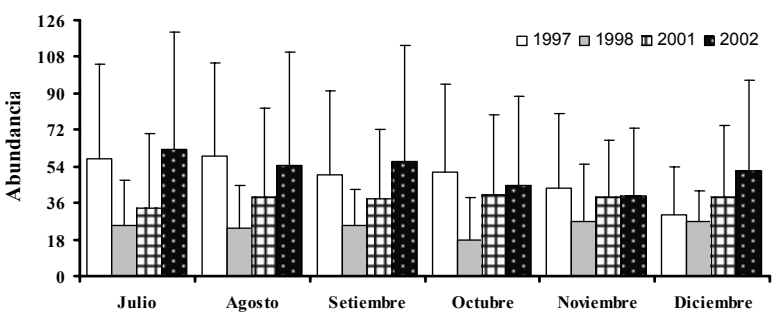

Figura 3. Promedios de abundancia en los años Niño y Niña con desviación estándar, donde se ve claramente la variación de los promedios entre los dos periodos de estudio.

Comparando los resultados de abundancia en los sustratos poblacionales de Amblyrhynchus cristatus (machos, hembras y juveniles) durante El Niño (199798) con los datos de los años de La Niña (2001-02), éstos muestran que si hay diferencia, principalmente entre la población de machos. Es evidente que este sustrato poblacional de adultos fue más afectado durante el fenómenos $(\mathrm{t}=2.195 ; \mathrm{gl}=5 ; \mathrm{P}<0.039)$. Se debe notar que la abundancia en los adultos machos aun no se ha recuperado en 2001-02 (Figura 4).

$\mathrm{La}$ abundancia de las iguanas hembras no fue muy afectada durante El Niño. Los valores fueron comparados con los de los años de La Niña y no fueron muy diferentes $(t=0.171 ; \mathrm{gl}=5 ; \mathrm{P}>0.44$; Figura 5).

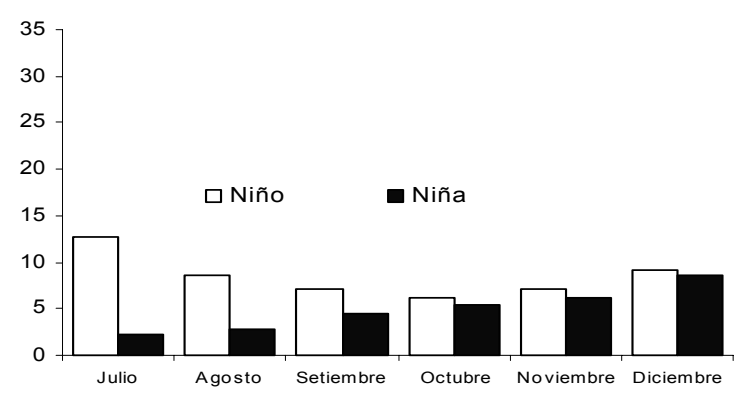

Figura 4. Abundancia de iguanas marinas machos durante El Niño (1997-98) vs. La Niña (2001-02).

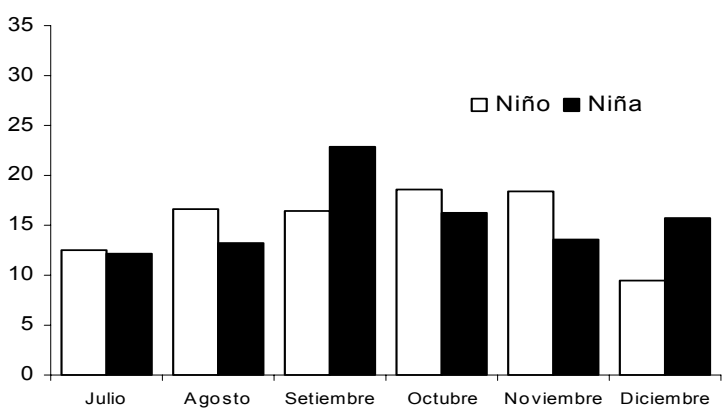

Figura 5 Abundancia de iguanas marinas hembras durante El Niño (1997-98) vs. La Niña (2001-02).

En El Niño la abundancia de los juveniles, fue muy afectada, pero en la Niña, la recuperación de la abundancia fue exitosa, por el reclutamiento de nuevos individuos cada año (Figura 6) comparadas las abundancias de ambos eventos muestran una marcada diferencia $(\mathrm{t}=7.377 ; \mathrm{gl}=5 ; \mathrm{P}<0.0004)$.

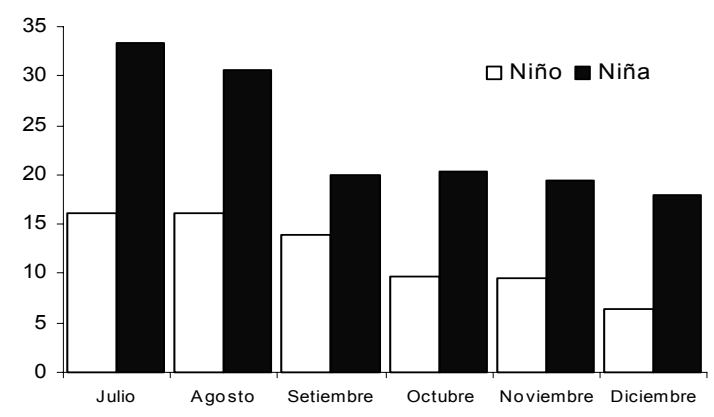

Figura 6. Abundancia de iguanas marinas juveniles durante El Niño (1997-98) vs. La Niña (2001-02).

\section{Discusión}

La abundancia promedio de A. cristatus al comienzo del evento El Niño (1997-98) se mantiene estable pero decrece notablemente en diciembre del mismo año, conforme con Castillo et al. (sin publicar) quienes mostraron que la salud de las iguanas marinas se deterioró a partir del sexto mes consecutivo con condiciones del evento El Niño (1997-98), es decir que el decrecimiento de la población de iguanas marinas está relacionado con las condiciones 
ambientales del momento. La abundancia en el periodo de La Niña no difiere luego de algunos meses (dependiendo de la prolongación del evento). Potenciales impactos son exacerbados en los animales adultos, produciéndoles disminución en peso y una posterior mortalidad, por la falta de algas verdes y rojas como alimento disponible en el ambiente inter y submareal a lo largo de las costas del archipiélago (Wikelski et al., 1993).

En lugares con pocas probabilidades de alimentación intermareal pero con oportunidades de alimentación submareal por buceo la mortalidad de los adultos fue pequeña y la juvenil muy grande. Por otra parte en sitios donde la alimentación es esencialmente intermareal, los animales jóvenes y adultos murieron en igual número durante El Niño (Laurie, 1984). Al relacionar los niveles de salud con el sexo de los animales se observó que durante el evento El Niño (1997-98) los machos adultos fueron los más afectados, presentando la mayor mortalidad (Castillo et al., sin publicar), las fuertes marejadas y un nivel de mar extremadamente alto, restringen el acceso a la zona de alimentación. Laurie (1984), observó una muerte en masa de las iguanas marinas en todas las islas. De igual forma en este estudio muestra claramente cómo la población de machos adultos sufrió una disminución drástica durante el evento El Niño 1997-98. Castillo et al. (sin publicar) comenta que el mayor deterioro del estado de salud detectado en machos adultos, con respecto a hembras y juveniles durante El Niño 1997-98, coincide con el patrón de mortalidad de el evento El Niño 1982-83.

La abundancia de las iguanas marinas en los estratos adultos, es recuperada luego de 4 años posteriores al fenómeno de El Niño (Laurie, 1986). En este estudio también es observado el mismo comportamiento anotado por Laurie $(1984,1986)$. Las Figuras 2, 5 y 6 muestran claramente la recuperación de la población de $A$. cristatus en el parche poblacional comprendido entre Punta Núñez y el muelle de la Estación Científica Charles Darwin; recuperación que también ha sido gradual en el lapso de 4 años. Según los estudios de Laurie (1986), Wikelski et al. (1993) y Wikelski \& Trillmich (1994), este estudio establece, luego de desarrollado un evento El Niño en las Islas Galápagos, que la recuperación de la abundancia en la población de iguanas marinas es lenta, entre 3 y 4 años. Este comportamiento también se refleja en el estudio realizado por Castillo et al. (sin publicar) en el que observa cómo la salud de las iguanas marinas es recuperada luego de un lapso de más o menos cuatro años de haber culminado el evento, por lo que está recuperación esta relacionada con la abundancia de estos animales.

Las condiciones de El Niño 1997-98 ocasionaron un claro efecto en la disminución de la abundancia por causas de mortalidad en los individuos adultos que reciben el mayor efecto por el evento. La recuperación de la población luego de El Niño es gradual; esto sugiere que las iguanas marinas son afectadas en su fisiología por estrés e ingestión de algas pardas, que son de difícil digestión, recurso utilizado por las iguanas en ausencia de las algas verdes y rojas de fácil digestión, según Wikelski \& Corinna (2000).

El decrecimiento de la abundancia en cada una de las estaciones de muestreo de la transecta, presentan disminución gradual durante el transcurso del evento El Niño. Durante los años subsiguientes de La Niña la abundancia de $A$. cristatus se recupera en forma gradual con grandes picos en algunas estaciones de muestreo, la misma que fue diferente entre ambos eventos, mostrando claramente que la población estuvo recuperada al cuarto año luego de transcurrido el fenómeno. Estas mismas observaciones fueron registradas por Laurie (1986) y Wikelski et al. (1993).

\section{Conclusión}

El fenómeno de El Niño es un evento natural que ocurre con frecuencia, en el Pacifico Sur. Su presencia altera y cambia las temperaturas de las aguas marinas y ambientales causando un deterioro en la flora y fauna marina que habitan en las costas del Océano Pacifico. Las iguanas marinas, fauna endémica de las islas Galápagos, son seres frágiles que son afectados por las anomalías del ambiente. El Evento ENOS 1997-98 causó un gran impacto en la abundancia de la población de $A$. cristatus disminuyendo su número en un $35.6 \%$ desde el inicio de El Niño (1997) hasta su finalización (1998). Las iguanas adultas, en especial los machos, fueron los más afectados durante el evento por ser las que buscan su alimento en zonas más profundas y alejadas de la costa. Cuatro años fueron requeridos para que la población de las iguanas marinas vuelva a recuperar los niveles normales de abundancia que tuvieron al inicio del evento. Pese a que los machos adultos de $A$. cristatus aun no muestran los niveles de abundancia de inicios del evento en 1997, se puede considerar recuperados los niveles de abundancia de la población, cuatro años después de iniciado el evento.

\section{Agradecimientos}

A la Fundación Charles Darwin, los donantes y administradores de los fondos, ya que sin su apoyo no hubiese sido posible la culminación de este trabajo Nuestra sincera gratitud, del mismo modo a cada uno de los voluntarios, becarios y empleados que acompañaron y apoyaron en la toma de los datos durante los seis años de estudio. También agradecemos a nuestros compañeros del Área de Investigación y Conservación Marina, en especial a Julio Delgado nuestro motorista, quienes apoyaron en la logística del trabajo en el campo. 


\section{Literatura citada}

Bartholomew G.A. \& Vleck D. 1979. The relation of oxygen consumption to body size and to heating and cooling in the Galapagos marine iguana, Amblyrhynchus cristatus. J. Comp. Physiol. B. Biochem. Syst. Environ. Physiol. 132: 285-288.

Cayot L.J. 1985. Effects of El Niño on giant tortoises and their environment. En G. Robinson \& E.M. del Pino, eds., El Niño en las Islas Galápagos: El Evento de 1982-1983, Fundación Charles Darwin, Quito.: 363-398.

Cooper J.E. \& Laurie W.A. 1987. Investigation of Deaths in Marine Iguanas (Amblyrhynchus cristatus) on Galapagos. J. Comp. Path. 97 (2): 129-136.

Laurie W.A. 1984. El Niño causa estragos nunca vistos en la población de iguanas marinas. La Sirena. 25: 11-14.

Laurie W.A. 1986. Effects of the 1982-83 El Niño sea warming on marine iguana (Amblyrhynchus cristatus Bell, 1825) populations on Galápagos. Report, CDRS Library. : 1-18.

Laurie W.A. 1990. Effects of the 1982-83 El NiñoSouthern Oscillation Event on marine iguana (Amblyrhynchus cristatus Bell, 1925) populations on Galapagos. In P. W. Glynn, ed., Global ecological consequences of the 1982-83 El NiñoSouthern Oscillation, Elsevier Oceanography Series. 52: 361-380.

Romero L.M. \& Wikelski M. 2001. Corticosterone levels predict survival probabilities of Galápagos Marine Iguana during El Niño events. Proceedings of the National Academy of Science (USA). 98: $7366-7370$.

Snell H.L. \& Márquez C. 2002. Iguanas Marinas. En: Reserva Marina de Galápagos. Línea Base de la Biodiversidad. Eva Danulat \& Grahm J. Edgar (editores). pp. 324-333. Estación Científica Charles Darwin/Servicio del Parque Nacional. Santa Cruz, Galápagos, Ecuador.

Wikelski M., Gall B. \& Trillmich F. 1993. Ontogenetic changes in food intake and digestion rate of the herbivorous marine iguana (Amblyrhynchus cristatus, Bell, 1825). Oecologia. 94: 373-379.

Wikelski M. \& Trillmich F. 1994. Foraging strategies of the Galápagos marine iguana (Amblyrhynchus cristatus): adapting behavioral rules to ontogenetic size change. Behaviour. 128 (3-4): 255-279.

Wikelski M. \& Corinna T. 2000. Marine Iguana Shrink to Survive El Niño. Nature. 403: 37-38.

\section{Apéndice de fotografías}

Fotos 1a-b. Iguanas marinas tomando sol en sus habitats naturales. a) En rocas adyacentes a los acantilados y b) En suelo arenoso, cerca de los manglares de la costa.

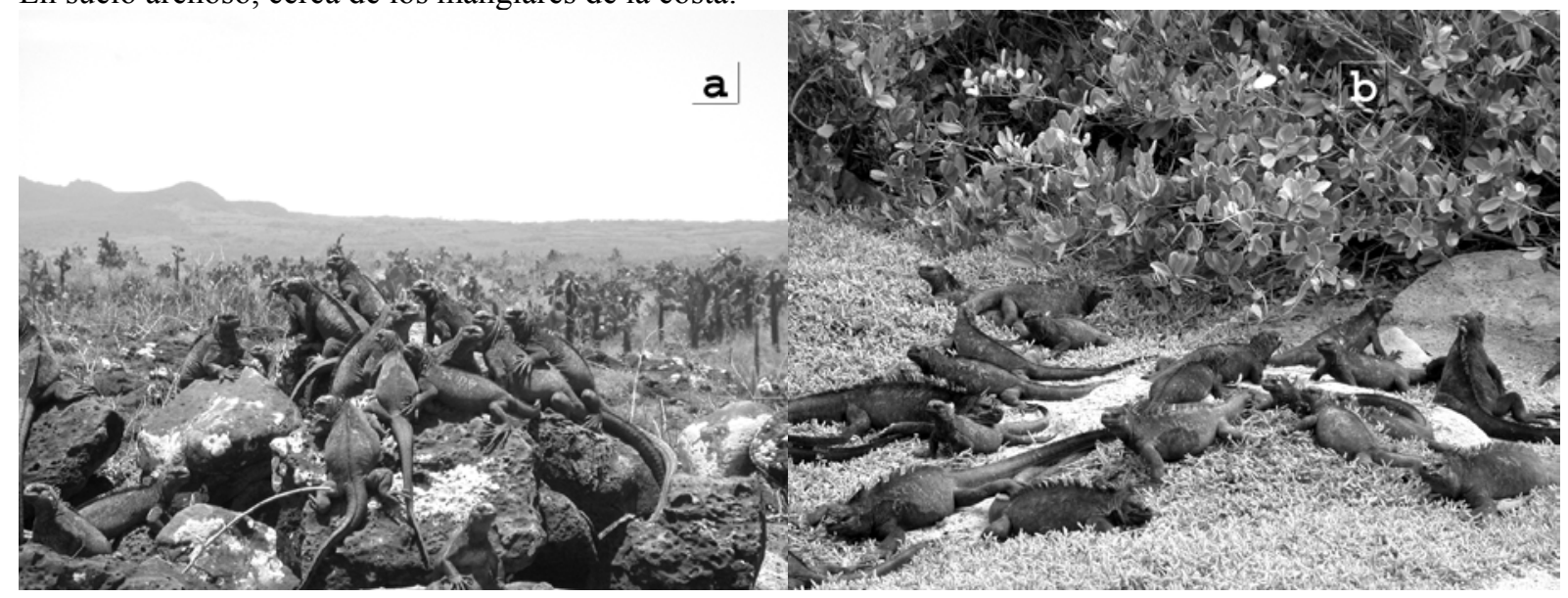

\footnotetext{
${ }^{1}$ Fundación Charles Darwin, Galápagos. Dirección postal: 17-01-3891 Quito, Ecuador. Correo electrónico: allerena@fcdarwin.org.ec

${ }^{2}$ Fundación Charles Darwin, Galápagos. Dirección postal: 17-01-3891 Quito, Ecuador. Correo electrónico: marquez@fcdarwin.org.ec

${ }^{3}$ Fundación Charles Darwin, Galápagos. Dirección postal: 17-01-3891 Quito, Ecuador. Correo electrónico: howard@fcdarwin.org.ec

${ }^{4}$ Fundación Charles Darwin, Galápagos. Dirección postal: 17-01-3891 Quito, Ecuador. Correo electrónico: albertoj@fcdarwin.org.ec
} 